\title{
HG-MAC: A Energy-Efficient Protocol for M2M Network
}

$$
\text { Chang } \mathrm{Xu}^{1, \mathrm{a}} \text {, Cong Wang }{ }^{2, \mathrm{~b}^{*}} \text {, Lingya } \mathrm{Liu}^{3, \mathrm{c}} \text {, and Ning } \mathrm{Li}^{4, \mathrm{~d}}
$$

\author{
1,2,3,4 Institute of Communications Engineering, PLA University of Science and Technology, Nanjing, \\ China \\ axuchangpla@sina.com, ${ }^{b}$ dolphin6488@163.com,
}

Keywords: medium access control (MAC), energy efficiency, Machine-to-Machine network, dormancy mechanism.

Abstract. The machine-to-machine network has become a leading solution in many important fields, such as battlefield surveillance, transport systems, industrial and agricultural automation. Typically, a M2M network consist of a large number of battery powered, concurrently access devices. Simplex MAC protocols can not adopt this situation. In this paper, a Hybrid and Grouping MAC (HG-MAC) is proposed. In HG-MAC, devices are grouped and graded to reduce collision and offer QoS guarantee. Through consisting of contention-based protocol and schedule-based protocol, devices can enter dormant state to improve energy-efficient. To improve flexibility, the duration of contention period and transmission period can be changed. The duration of transmission period is decided by the contention period length, thus the contention period length is a significant factor to the network performance. An analysis of the relationship between the contention period length and the average transmission rate is made in this paper. The simulation results demonstrate the effectiveness of the proposed HG-MAC protocol.

\section{Introduction}

Machine-to-Machine (M2M) Communication is defined as the information exchanged between machines and machines without any human interaction, it expected to grow exponentially in many fields such as battlefield surveillance, transport systems, industrial and agricultural automation etc. [1]. Like in all shares-medium networks, medium access control is the foundation of the network successful operation. There are many differences between MAC protocols in M2M networking and traditional ones. Thus it is very necessary to design a new MAC protocol for M2M networks. To design a good MAC protocol for M2M networks, we have considered the following attributes. The first is the energy efficiency. A major power consuming component of a device is the radio, which is controlled by the MAC protocol. Most of the M2M networks are constituted of devices which are battery powered and difficult to change or recharge batteries. In fact, the devices are disposable in more and more application scenes. The second important attribute is collision. There are tremendous number of devices in service coverage and concurrent network accessing attempt from these devices. Collisions will cause energy waste, sending fault etc.. Reducing collision will improve the performance of network. The third attribute is flexibility. The traffic load may change over space and time in M2M networks. A good MAC protocol should accommodate such network change. Based on this, the present paper proposes HG-MAC protocol for M2M networks which combines the benefits of both contention-based protocol and reservation-based protocol. It reduces energy consumption through dormancy mechanism. It uses grouping and hierarchy to alleviate collision. To increase the flexibility, the frame time is allowed to change over time.

The remainder of this paper is organized as follows. We introduce the related work in Section II. In section III, we describe the system setting and assumption. Then, the design of HG-MAC is proposed in section IV. In section V, we analyze the relation of throughput and the duration of COP. Performance study and evaluation are given in Section VI. Section VII concludes the paper. 


\section{Related Work}

When developing new protocol, it is necessary to investigate large amount of previous studies thoroughly. There is no unified MAC protocol classification in current researches. In a general way, MAC protocols can be classified into three branches based on their medium access strategies: contention-based protocol, limited-contention protocol and schedule-based protocol or based on synchronization request: global synchronization protocol, local synchronization protocol and asynchronous protocol [2]. The contention-based protocol allows devices access to medium randomly. It is unnecessary to keep synchronous, thus a contention-based protocol is always an asynchronous protocol. The advantages of contention-based protocols are flexibility, practicable and low overhead. But when the traffic load is heavy, performance shall become very poor due to collision. The typical contention-based protocol is CSMA/CA. The limited-contention protocols limit the accessing devices number in the same time. To tackle this issue, grouping and time division are normal solution. It requests devices in one group or time slice keeping synchronous. Thus a limited-contention protocol is also a local synchronization protocol. The key factor of limited-contention protocols is taking a tradeoff according to the application scene. The schedule-based protocol often adopts the mechanism of duty cycle which means devices alternating between active and sleep states. All devices change states in a strict time sequence in schedule-based protocol. It requests synchronization of all devices. Thus, a reservation-based schemed can be regarded as a global synchronization protocol. The mechanism of duty cycling can make it very easy and efficient to schedule dormancy, thus it is good for energy efficiency. There is no collision in a reservation-based protocol, it mean that the network perform very well with heavy traffic load. The major disadvantages are that the channel utilization is low when the load of network is low and flexibility is very poor. And synchronization request increases the cost of network. MAC protocols also can be classified by which initiates the transmission. Transmission can be initiated by the sender like CSMA-CA [3], or by the receiver like RC-MAC [4]. In receiver initiated MAC, the receiver broadcasts a beacon to announce that it is ready to receive. The sender will receive the beacon and start to transmit. Receiver initiated MAC can save energy because the receiver can be into dormant stat. In RMAC [5], time is divided into three periods: SYNC, DATA, and SLEEP. A control frame called Pioneer Frame (PION) is forwarded by multiple hops in SYNC. The PION informs the device on a routing path of when it wakes up and sleeps. The PION also serves as a RTS and CTS. In DATA period, the data message is send. In TreeMAC [6], time is divided into cycles and each cycle is divided into $\mathrm{N}$ frames of 3 slots. Nodes of the same depth on the tree are assigned different frames for transmission. In a frame, the transmission slot is calculated as (L-1) mod 3 where $L$ is the depth of the node. Using three slots, a node can avoid contention with its previous and next hop. Z-MAC [7] combines CSMA and TDMA to increase channel utilization, a node is allowed to contend a slot if it is not used. To further increase the channel utilization, time frame rule is designed to adapt the local frame size to a nodes local neighborhood size.

\section{System Setting and Assumption}

As showed in Fig. 1, we consider a M2M networks with one CN (center node) and $N$ number of DNs (data node). The network is dominated by the CN. All DNs collect data and send them to CN. All data messages have same size. DNs are graded into two priority level. The nodes in high priority level can contend access opportunity in every frame. The nodes in common priority level have same contention probability and it equals to $P$. DNs in common priority level are separated into many groups. Every group has a unique group number to distinguish with other groups. The $\mathrm{CN}$ can communicate with all DNs by one hop. In order to simplify the model, we do not consider the mistake in the transmission. 


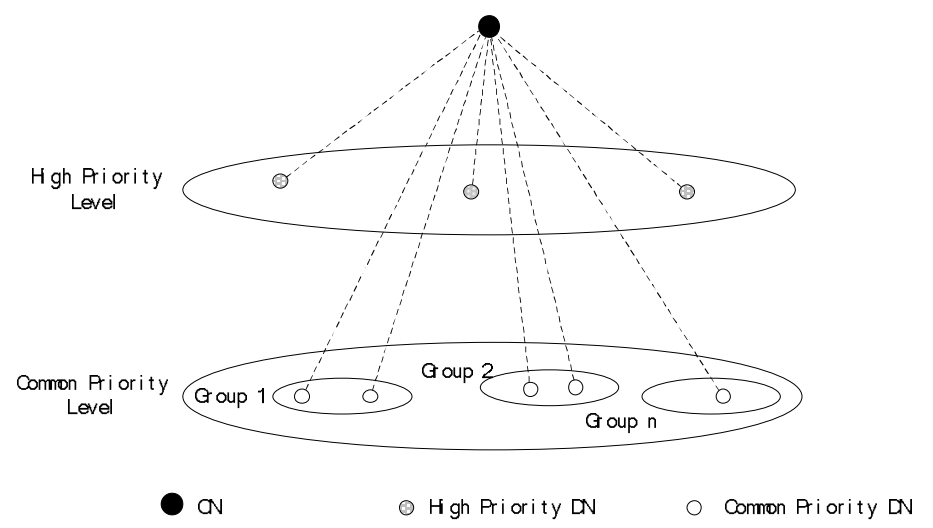

Fig. 1. Topology

\section{The Design of HG-MAC Protocol}

In this section, HG-MAC protocol is proposed for the M2M networks. The whole process of transition is separated into many frames which include four periods: Notification Beacon Period (NBP), Contention Only Period (COP), Transmission Beacon Period (TBP), and Contention Free Period (CFP), as shown in Fig. 2. The duration of frame is probable different and it is decided by the length of COP period. The particular description of each period is given as follows:

Notification Beacon Period (NBP): $\mathrm{CN}$ broadcasts the notification beacon to all DNs at the beginning of the frame. The notification beacon pronounces the start of the frame. It includes the duration of COP and the group number of DNs group can contend the transmission opportunities. DNs in the group ready to contend the opportunities and the other devices are dormant in the COP period.

Contention Only Period (COP): In this period, DNs in the allowable group contend the transmission opportunities based on CSMA/CA method. The device send request message to the center node. If the $\mathrm{CN}$ is succeed to receive the request message, it will increase a counter by one and send an ACK message to this device. The ACK includes the counter of CN. Because every data message has the same length, DNs can easily calculate the transmission time in the CFP. DNs enter the state of dormancy after receive the ACK and wake up in the end of COP to receive TBP. The devices in groups which are not allowed to transmit will be dormant in COP, and wake up in the end of COP to receive Transmission Beacon. In the M2M network some DNs are important, their data require high priority and lower delay. Thus in HG-MAC, we allow these DNs without group and they can contend transmission opportunities in every COP.

Transmission Beacon Period (TBP): In this period, the center node broadcasts transmission beacon to all devices. The transmission beacon includes the duration of CFP which is calculated using the counter. All devices wake up in this period to receive transmission beacon.

Contention Free Period (CFP): In this transmission period, the devices transmit data based on TDMA method. The waking up time of DNs include the NBP duration, the time it contends chance in COP and the transmitting time in CFP. The waking up time of other devices only includes NBP and TBP.

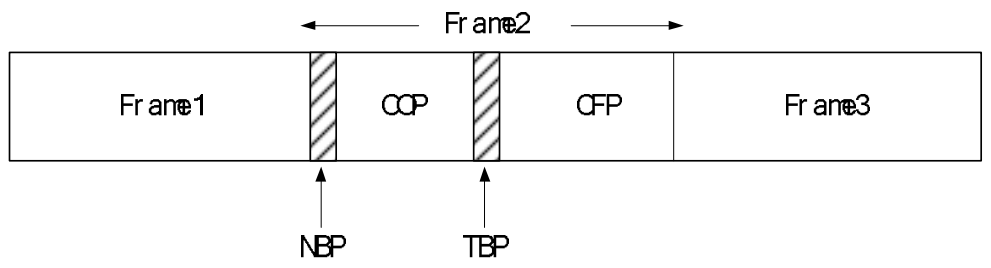

Fig. 2. The frame structure 


\section{Relationship of Data Transmission Rate (DTR) and Duration of COP}

In HG-MAC, the duration of COP is a very important parameter. Frame length and CFP length are closed related to it. Data Transmission Rate is an import performance of MAC protocol. Thus, we derive the relationship of the duration COP and the DTR in this section. Here, we define $P$ as the data transmission rate, which is described as:

$$
P=\frac{m D}{T_{C O P}+m T_{\text {tra }}+T_{\text {bea }}}
$$

where $m$ is the number of devices which successful get the chance to send message in the COP and D is the data size sent to the center node. Let $T_{C O P}, T_{\text {tra }}$ and $T_{b e a}$ denote the duration of COP, the duration of CFP and the sum duration of sending beacon messages.

Here, let $T_{i}$ denote the time between $(i-1)$ th and $i$ th successful contention. Since $T_{C O P}$ is the sum of random variable $T_{i}(i=1, \ldots, m)$ as Eq. 2 .

$$
T_{C O P}=\sum_{i=1}^{m} T_{i}
$$

In the COP, collisions and idles are alternately in $T_{i}$. There are $N_{i}^{c}$ number of collisions between $(i-1)$ th and $i$ th successful contention. idle $_{i, j}$ is the $j$ th idle time and coll $_{i, j}$ is the $j$ th collision time in $T_{i} . S_{i}$ is the sum time of sending ASK and ACK. Then, we have Eq. 3 and Eq. 4.

$$
\begin{gathered}
T_{i}=\sum_{j=1}^{N_{i}^{c}}\left(\text { idle }_{i, j}+\operatorname{coll}_{i, j}\right)+i d l e_{i, N_{i}^{c}+1}+S_{i} \\
T_{C O P}=\sum_{i=1}^{m}\left[\sum_{j=1}^{N_{i}^{c}}\left(i d l e_{i, j}+\operatorname{coll}_{i, j}\right)+i d l e_{i, N_{i}^{c}+1}+S_{i}\right]
\end{gathered}
$$

To obtain the close-form expression of $T_{C O P}$ and $P$. Due to the independent variable $T_{i}$ [8], we use average number to replace them. We have Eq. 5 and Eq. 6.

$$
\begin{gathered}
E\left[T_{C O P}\right]=\sum_{i=1}^{m} E\left[T_{i}\right] \\
E\left[T_{C O P}\right]=m\left\{\left(E\left[N_{i}^{c}\right]+1\right) E[\text { idle }]+E\left[N_{i}^{c}\right] E[\text { coll }]+E[S]\right\}
\end{gathered}
$$

Every DN sends messages with invariant probability $p$ in a time slot. For a given number of devices $A$, we have Eq. 7 and Eq. 8 according to [9]:

$$
E\left[N_{i}^{c}\right]=\frac{1-(1-p)^{A-i}}{(A-i) p(1-p)^{A-i-1}}-1
$$




$$
E\left[\text { Idle }_{i}\right]=\frac{(1-p)^{A-i}}{1-(1-p)^{A-i}} \delta_{i d l e}
$$

When $A \square i$, we can have Eq. 9 and Eq. 10.

$$
\begin{gathered}
E\left[N_{i}^{c}\right]=\frac{1-(1-p)^{A}}{A p(1-p)^{A}}-1 \\
E\left[\text { Idle }_{i}\right]=\frac{(1-p)^{A}}{1-(1-p)^{A}} \delta_{\text {idle }}
\end{gathered}
$$

Substitute Eq. 9, Eq. 10 into Eq. 6, we can have Eq. 11.

$$
m=\frac{T_{C O P}}{\left(\frac{1-(1-p)^{A}}{A p(1-p)^{A}}\right) E[\text { idle }]+\left(\frac{1-(1-p)^{A}}{A p(1-p)^{A}}-1\right) E[\text { coll }]+E[S]}
$$

We use $\sigma$ to replace $A, P, E[$ idle $], E[$ coll $]$ and $E[S]$, we can have Eq. 12 .

$$
\sigma=\left(\frac{1-(1-p)^{A}}{A p(1-p)^{A}}\right) E[i d l e]+\left(\frac{1-(1-p)^{A}}{A p(1-p)^{A}}-1\right) E[\text { coll }]+E[S]
$$

Thus, we can obtain the simplified expression of relationship between $m$ and $T_{C O P}$ as Eq.13.

$$
m=\frac{T_{C O P}}{\sigma}
$$

Hence, we can get the simplified expression of relationship between $P$ and $T_{C O P}$ as Eq. 14 .

$$
P=\frac{T_{C O P} D}{\sigma T_{C O P}+T_{C O P} T_{\text {tra }}+\sigma T_{\text {bea }}}
$$

\section{Performance and Evaluation}

To evaluate the performance of HG-MAC, several simulations are carried out using the OPNET in the following section. HG-MAC is compared with the CSMA/CA and TDMA in terms of data transmission rate, utility and energy efficiency. Finally, we research the relationship of COF and DTR with several simulations. The simulation topology is a 301-node star network which 300 DNs communicated to one $\mathrm{CN}$. The main parameters used by simulation are provided in Table 1 . The most of simulation parameters is derived from IEEE 802.11 standard. 
Table 1. The Simulation Parameters

\begin{tabular}{|l|l|l|}
\hline$D_{n b}$ & $160 \mathrm{bit}$ & The Notification Beacon size. \\
\hline$D_{t b}$ & $192 \mathrm{bit}$ & The Transmission Beacon size. \\
\hline$D_{a s k}$ & $192 \mathrm{bit}$ & The ASK packet size. \\
\hline$D_{a c k}$ & $192 \mathrm{bit}$ & The ACK packet size. \\
\hline$D_{\text {data }}$ & $6000 \mathrm{bit}$ & The Data size. \\
\hline$T_{c o p}$ & $0.003 \mathrm{sec}$ & The duration of COP \\
\hline R & $1.7 \mathrm{Gbps}$ & The data rate. \\
\hline SIFS & $28 \mu \mathrm{s}$ & $\begin{array}{l}\text { The duration of short interface } \\
\text { space. }\end{array}$ \\
\hline DIFS & $128 \mu \mathrm{s}$ & $\begin{array}{l}\text { The duration of data interframe } \\
\text { space. }\end{array}$ \\
\hline Slot Time & $50 \mu \mathrm{s}$ & The duration of slot time. \\
\hline
\end{tabular}

\section{A. Data Transmission Rate (DTR)}

In this paper, the data transmission rate is the rate of useful data collected by the central node. We compare the DTR between the HG-MAC protocol, CSMA/CA and TDMA in terms of the different load. The variation of traffic load is represented by the change of attempt times in a fix interval. The attempt times is the average messages which DN sends in a fix interval and it can be a decimal. We can obtain the mathematical expression of data transmission rate as Eq. 15.

$$
D T R=\frac{D}{T_{\text {sim }}}
$$

where $D$ is the sum size of useful data collected by the central node and is fixed to the size of 1000 data packets. $T_{\text {sim }}$ is the simulation time which completes the data collection. To achieve an exact conclusion, two different intervals with $0.004 \mathrm{sec}$ and $0.2 \mathrm{sec}$ are used to compare. The horizontal ordinate is the Attempt Times and the vertical ordinate is the DTR in Fig.3 and Fig.4. In Fig.3, when the interval equal to 0.004 , the performance of TDMA is better than the others and the HG-MAC performed moderately. The DTR of TDMA and HG-MAC are increased with the Attempt Times raised. The CSMA/CA is the worst because of collision. With the Attempt Times raised, the DTR of CSMA/CA is decreased because the conflict is more intense. Fig. 4 clearly demonstrates that the HG-MAC can achieve a higher DTR in the most of the region when the traffic load is middle.

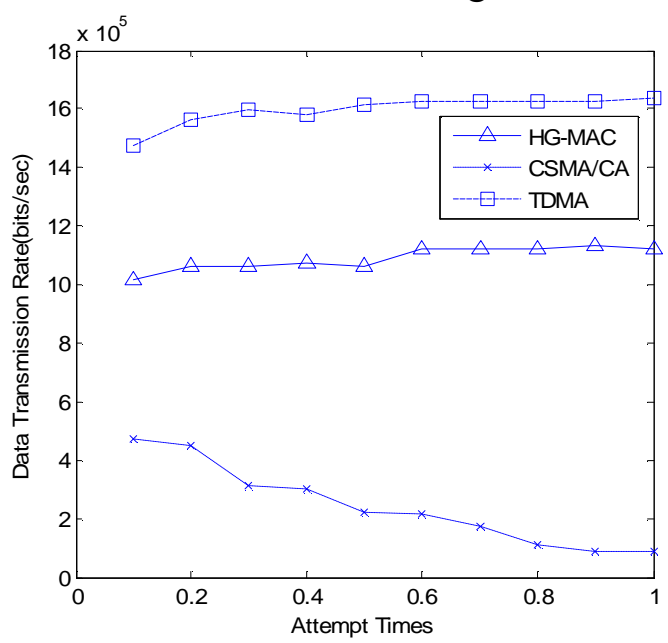

Fig. 3. DTR in heavy traffic load 


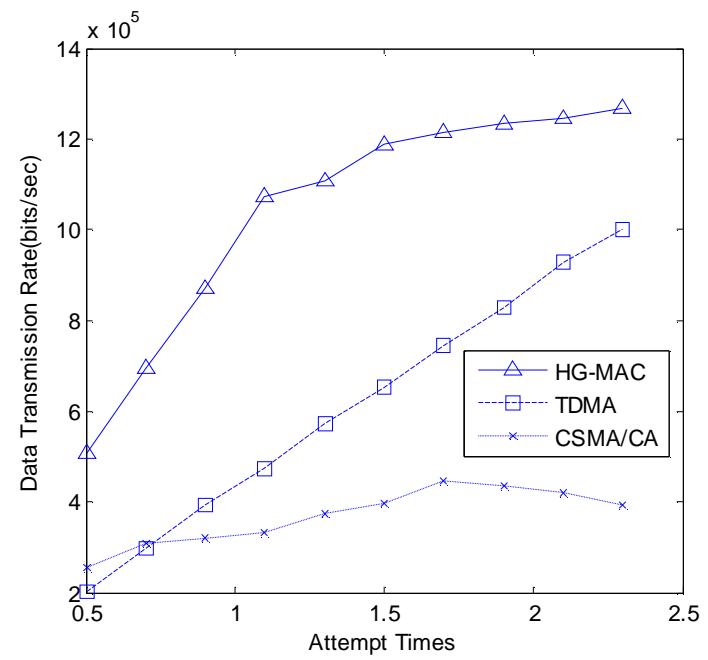

Fig. 4. DTR in middle traffic load

\section{B. Energy Efficiency (EE)}

In this paper, we define the energy efficiency as the reciprocal of all data nodes consuming energy which is the central node receiving a fixed number of data packets. According to [10], the energy consumption ratio of transmission, receiving, idle listening and dormancy is 15:13:13:1. Let ce denote the energy consumed to send packets and $\alpha$ denote the normalized coefficient. In this simulation, $\alpha$ equals to 10000 . We have:

$$
E E=\frac{\alpha}{c e}
$$

In Fig. 5, the horizontal ordinate is the Attempt Times and the ordinate is the normalized energy efficiency that central node receives 1000 data packets. The interval of generate data is 0.2 sec. Because the dormant mechanism, the energy efficiency of HG-MAC is higher than others and its variation is tiny with the change of load. Due to that the node shall wake up to listen to the channel, the energy efficiency of TDMA raises with the increase of traffic load. The energy efficiency of CSMA/CA is lower than others and declines with the decrease of traffic load when the traffic load is heavy.

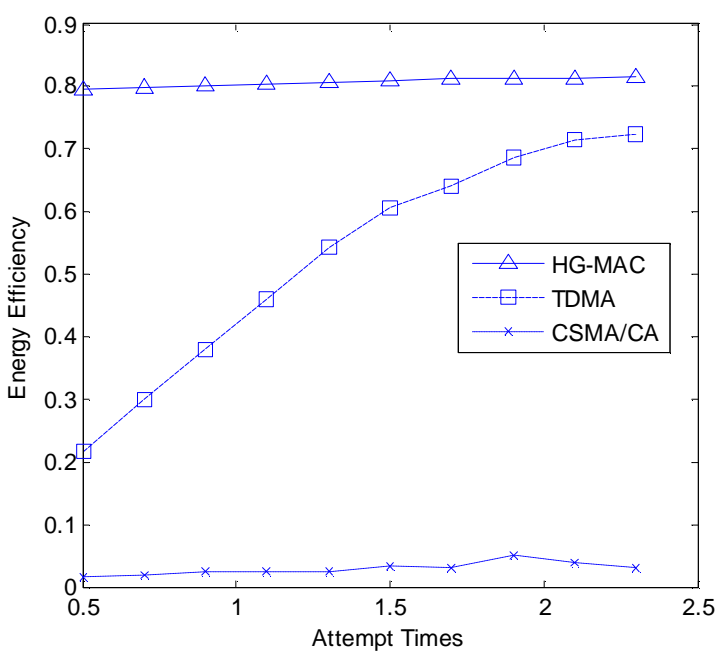

Fig. 5. Energy efficiency versus the attempt times

\section{Channel Utility $(C U)$}

In this paper, we define the channel utility $(\mathrm{CU})$ is the ratio of the time channel be used to the total simulation time. Let $T_{t s}$ denote the time of channel be used to transmit and $T_{\text {sim }}$ denote the simulation time. We have the utility is showed as Eq. 17. 


$$
C U=\frac{T_{t s}}{T_{s i m}}
$$

In Fig. 6, the horizontal ordinate is the attempt times in an interval $0.2 \mathrm{sec}$ which indicates the variation of traffic load and the ordinate is the channel Utility in terms of the hybrid protocol, CSMA/CA and TDMA. The generation interval is $0.2 \mathrm{sec}$. It can be seen from Fig. 6 that the Channel Utility of HG-MAC is higher than the others in the middle traffic load condition.

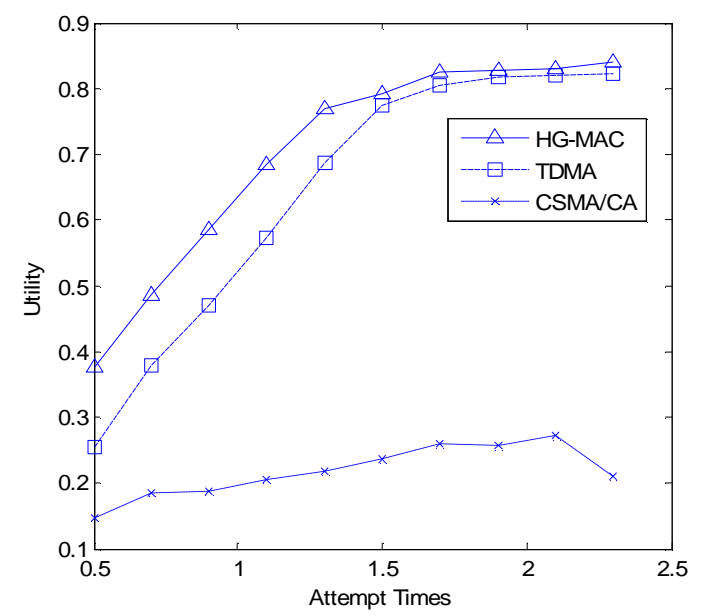

Fig. 6. Channel utility versus the attempt times

\section{DTR and the duration of COP}

In this subsection, several simulations are carried out to probe the relationship of the DTR and the duration of COP. In Fig.7 and Fig.8, the horizontal ordinate is the duration of COP and the ordinate is the DTR. To illustrate the relationship, we also compare the data transmission rate in term of different attempt times. The generation interval is $0.2 \mathrm{sec}$ in this simulation. Every simulation data is the average value of 20 simulation results. Fig. 7 and Fig. 8 show that the relationship of COF and DTR when the attempt times equals to $0.3 \mathrm{sec}$ and $1 \mathrm{sec}$. The duration of COP which makes the DTR highest is 0.004 sec and $0.0044 \mathrm{sec}$ in the situation in Fig. 7 and Fig. 8. The simulation result indicates that an optimal duration of COP which makes the DTR maximum is different in terms of traffic load changed. Thus it is very important to find a best duration of COF according to the traffic load.

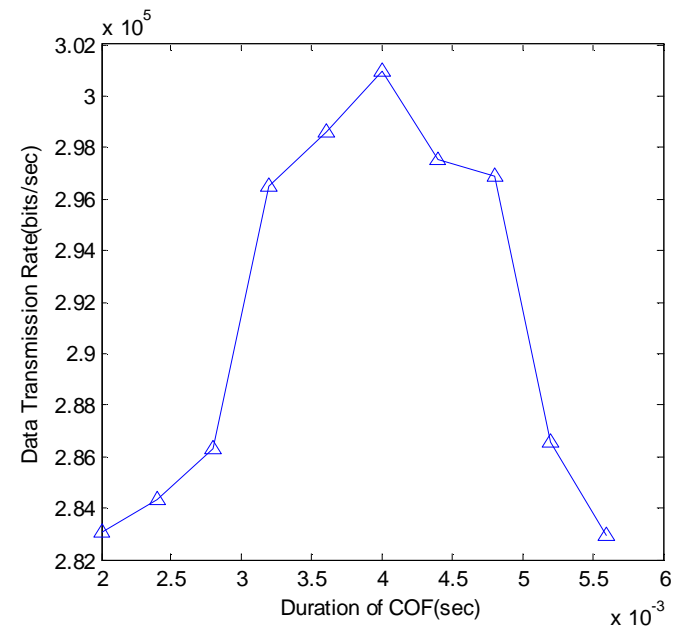

Fig. 7. The relationship of COP and DTR with attempt times $=0.3$ 


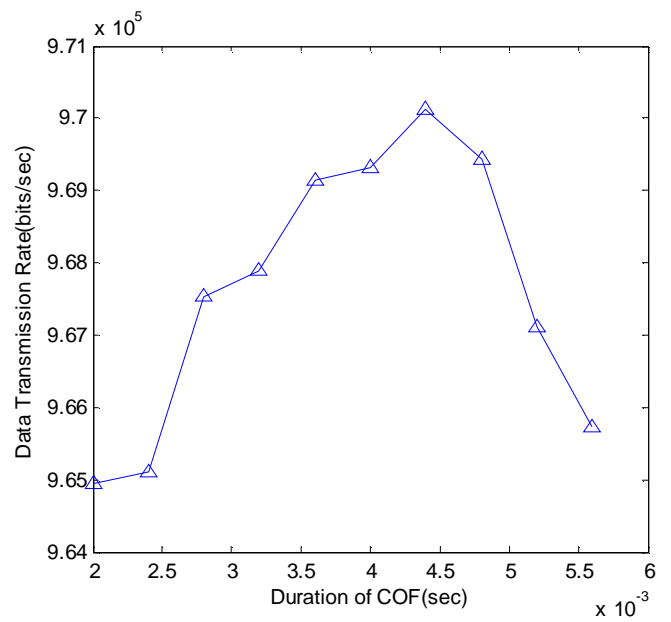

Fig. 8. The relationship of COP and DTR with attempt times $=1$

\section{Conclusions}

This paper formulates HG-MAC protocol for data gather operation M2M networks. In our scheme, contention-based protocol and schedule-based protocol are combined together. Each frame is divided into NBP, COP, TBP and CFP periods. The data nodes (DNs) contend the transmission opportunities in the COP period and send data in CFP period. In order to decrease the collision, data nodes are divided into groups. In order to guarantee the QoS, DNs are hierarchical. A dormant mechanism is designed to decrease energy consuming. In HG-MAC protocol, the duration of COP is closely related to the data transmission rate, and we give a mathematical analysis. Simulation results clearly show that the HG-MAC performs better than CSMA/CA and TDMA in terms of data transmission rate, utility and energy efficiency. In the M2M networks, the traffic load is variable with the change of time and space. The duration of COP in this simulation is constant. In the future, a self-adaption and learning scheme which adjust COP should be considered to improve the flexibility.

\section{Acknowledgment}

This work was supported by Major Research Plan of National Natural Science Foundation of China (No. 91438115); Jiangsu Province National Science Foundation (BK2011002); National Natural Science Foundation of China(No. 61371123).

\section{References}

[1] I. F. Akyildiz, W. Su, Y. Sankarasubramaniam and E. Cayirci, A survey on sensor networks. IEEE Communications Surveys Tutorials 40 (2002) 102-114.

[2] Pei Huang, Li Xiao, Matt W. Mutka and Ning Xi. The Evolution of MAC Protocols in WirelessSensor Networks: A Survey, IEEE Communications Surveys Tutorials 15 (2013) 62-64.

[3] G. Wang, X. Zhong, S. Mei and J. Wang, An Adaptive Medium Access Control Mechanism for Cellular Based Machine to Machine (M2M) Communication, IEEE ICWITS (2010)1-4.

[4] P. Huang, C. Wang, L. Xiao and H. Chen, RC-MAC: A receiver-centric medium access control protocol for wireless sensor networks, IEEE Transactions on 64 (2010)1-9.

[5] S. Du, A. K. Saha and D. B. Johnson, RMAC: A routing-enhanced duty-cycle MAC protocol for wireless sensor networks, IEEE International Conference on Computer Communication 174 (2007) $1478-1486$. 
[6] W. Z. Song, R. Huang, B. Shirazi and R. LaHusen, TreeMAC: Localized TDMA MAC protocol for real-time high-data-rate sensor networks, Pervasive Computing and Communications (2009)1-10.

[7] I. Rhee, A. Warrier, M. Aia and J. Min, Z-MAC: a hybrid MAC for wireless sensor networks, IEEE/ACM Transactions on 16 (2005)90-101.

[8] R. Bruno, M. Conti and E. Gregori, Optimal Capacity of p-Persistent CSMA Protocols, IEEE Communication Letters 7 (2003)139-141.

[9] F. Cali, M. Conti and E. Gregori, Dynamic IEEE 802.11: design, modeling and performance evaluation, IEEE Communications Surveys Tutorials 18 (2000)1774-1786.

[10] Estrin D, Wireless Sensor Networks Tutorial Pan IV: Sensor Network Protocols, Mobicom. (2002)23-28. 